\title{
AVALANCHES IN NETWORKS OF WEAKLY COUPLED PHASE-SHIFTING ROTATORS*
}

\author{
A.YU. SHAHVERDIAN ${ }^{\dagger}$ AND A.V. APKARIAN ${ }^{\ddagger}$
}

\begin{abstract}
The networks of phase-shifting rotators interacting through exchange of weak $\delta$-kicks are considered. Such a rotator consists of a particle rotating on a circle which at some discrete moments receives some $\delta$-kicks. We assume that the kicks are not of mechanical character: they change a particle's position but not the rate. A comparison of the rotator networks with the BTW model of self-organized criticality, Burridge-Knopoff's model in seismicity, Herz-Hopfield neural networks, and the Turing-Smale system in biological cells is presented. This work studies the avalanches in rotator networks - the events when a number of rotators almost simultaneously hit some threshold levels. The asymptotic relations linking distribution of avalanches with the architecture of a network are proved. The equivalence of two well-known power-law conjectures, in lattice models of statistical physics and in interacting threshold systems, is established.
\end{abstract}

Key words. dynamical networks, self-organized criticality, avalanches

PACS: 05.45.Xt.

\section{Introduction}

Let us explain our abstract and describe the networks of threshold microsystems considered in this work; the formal definitions are given in the following sections. Let $E_{N}=[1,2, \ldots, N]$ be the sequence of $N \geq 2$ integers and $\bar{\omega}=\left(\omega_{1}, \ldots, \omega_{N}\right) \in \mathbb{R}^{N}$ be a vector with non-zero components. Let $\mathcal{R}=\left\{R_{1}, \ldots, R_{N}\right\}$ be a set of $N$ rotators $R_{i}$ (some of them may coincide): the $R_{i}$ consists of a circle $C_{i}$ and a particle $P_{i}$ rotating on it with constant angular rate $\left|\omega_{i}\right|$; rotation occurs clockwise or anti-clockwise depending on whether $\omega_{i}$ is positive or negative. Let to every $i \in E_{N}$ a label $\rho_{i} \in C_{i}$ as well as a nonempty set of indices $\sigma_{i} \subset E_{N}, i \notin \sigma_{i}$ be prescribed: $\rho_{i}$ is understood as some threshold position for the motion of $P_{i}$ and $\bar{\sigma}=\left(\sigma_{1}, \ldots, \sigma_{N}\right)$ assigns the neighborhood in the network $\mathcal{R}: R_{i}$ interacts with $R_{j}$ iff $j \in \sigma_{i}$. The interaction is as follows: each time when $P_{i}$ hits the level $\rho_{i}$, each $P_{j}$ whose index $j$ is in $\sigma_{i}$ instantaneously rotates (shifts) along its orbit on $C_{j}$ by a given small angle (the intensity of interaction). The following is required: a shifting of a particle does not affect its rate (it remains the same before and after interaction) and the moments when the particle hits its threshold are separated by a positive constant. This work studies the avalanches in such rotator networks - the events when a number of rotators in the network almost simultaneously attain their corresponding thresholds.

We claim that if in a system of almost identical rotators the interaction of small intensity is included, then provided certain extent in couplings there arises a dynamics which is complicated. The situation is reminiscient of Turing's statement [20, 22] in biological cells: Turing-Smale's theorem says that when put into conditions allowing certain interaction, the system of initially inert cells begins to exhibit nontrivial oscillatory dynamics. It can be said that we study the Turing-Smale statement adapted to the framework of self-organized criticality (SOC), a theory resulting from numerous studies in the BTW model (a description is given below) which deals with large

*Received: October 28, 2007; accepted (in revised version): December 30, 2007. Communicated by David Cai.

${ }^{\dagger}$ Corresponding author: Institute for Informatics and Automation Problems, Armenian National Academy of Sciences, Paruir Sevak Str., 1, Yerevan, 0014, Armenia (svrdn@yerphi.am).

${ }^{\ddagger}$ Department of Physiology, Northwestern University, The Medical School, 303 East Chicago Ave., Tarry Bldg. 5-703, Chicago, Illinois, 60611 USA (a-apkarian@northwestern.edu). 
systems of interacting threshold elements and where the notion of avalanche is a basic one. Indeed, in both systems the interaction is such that it always tends to equalize the current states of microsystems, but in either case the system's global dynamics appears to be of oscillatory or even unpredictable character. It is an important advantage of considering rotator networks (RN) that despite the fact that the microdynamics in these systems is close to microdynamics usually accepted in SOC, they permit theoretical analysis - this circumstance is due to a justification of the definition of an avalanche and some restrictions imposed on the network's parameters. Thus, our statement permits a rigorous answer to Smale's question about the role of connectivity in interacting systems [20].

The sandpile- or BTW model (named after the authors who designed the concept of self-organized criticality, Bak, Tang, and Weisenfeld [2]), a typical SOC system, is the following (the details are omitted; for formal definition see [6]): at each moment of discrete time a particle from some external source at a randomly chosen site (node) of a given $d$-dimensional finite lattice is added; if at any site the number of particles exceeds $2 d$ they are uniformly distributed among the nearest neighbors; if because of a large number of particles in the system this procedure cannot be continued, some of them are removed to a "sink" and the entire procedure is repeated again. This system inherits some dynamical features of Burridge-Knopoff's (BK model) "earthquake machine" (a set of sliding blocks connected by springs; [4], see also [1]) — in both systems avalanches of arbitrary size occur in an unpredictable way and according to simulations $[2,4]$ their distribution follows the power law:

$$
P(k) \sim \text { const } \cdot k^{-\lambda_{1}}, \quad\left(\lambda_{1}>0\right),
$$

where $\mathrm{P}(\mathrm{k})$ is the frequency with which the avalanches of size $k$ take place (an avalanche of size $k$ is an event where at some $k$ different sites the number of particles at each of them simultaneously becomes equal to the threshold $2 d$ ). Since Equ. (1.1) is well consistent with Gutenberg-Richter's fundamental law in seismology (empirical distribution of earthquakes' intensity), these two systems were recognized as valuable means in modelling of seismic events. Later, numerous applications of SOC in various areas were proposed. In what follows $\mathrm{RN}$ is compared with a neurosciencerelated system which we refer to as the Herz-Hopfield model (the HHM; should not be mixed with the other well known Hopfield model) suggested in $[11,12,13]$ on neural coherent firing. In contrast to many works in SOC that mostly deal with numerical simulation, the work of these authors contains rigorously proven results. We also briefly discuss the Turing-Smale mathematical model of morphogenesis: despite this system being given in the form of ODEs, one can notice its common features with $\mathrm{RN}$, whose dynamics are described in terms of thresholds and avalanches.

Our interest is concentrated on avalanches and their distribution; in neural networks an avalanche of size $k$ is treated as a simultaneous firing of an assembly of $k$ neurons, in BK- and BTW models it associates with an earthquake of intensity $k$. Every avalanche of size $k$ is followed by (almost) simultaneous interaction of some $k$ microsystems, and hence we study the intensity of interaction in the network. In these systems a threshold nature phenomenon is modelled in such a way that the emergence of an avalanche is associated with the attainment of the system's orbit of the boundary of state space (e.g., if this space is the $N$-dimensional unit cube $\mathbb{D}^{N}=[0,1]^{N}$, every attainment of its any $k$-dimensional, $1 \leq k \leq N$ edge can be understood as an avalanche of size $k$ ). The method used in the present work is the same as in [17] and is based on Weyl's theorems in uniform distribution [14]. This theorem is applied 
as follows: with every avalanche of size $k$ we associate an $N$-dimensional region in state space $\mathbb{D}^{N}$ which occupies some neighborhood of $k$-dimensional edges; then the network's orbits are approximated by the orbits of a simpler system where the Weyl theorem holds, and thus the computation of the frequencies of avalanches is reduced to a simpler task of computation of volumes of such regions.

This work contains a generalization of our earlier results from [17]. More attention to possible applications in neuroscience is given. The theorems presented are of asymptotic character: they are valid when some parameters of the network are small. In Section 2 we define phase-shifting rotators, rotator networks RN, and avalanches and present their comparison with the corresponding notions from other works. In Section 3 we consider RN assigned on Euclidean lattices: Theorem 3.5 relates to frequency of avalanches which occur over a given set of nodes of the lattice; Theorems 3.6 and 3.8 establish the formulas connecting dynamical (avalanche distribution) and combinatorial characteristics of the network; Corollary 3.9 states that in $\mathrm{RN}$ the power-law conjecture (1.1) for distribution of avalanches is almost equivalent to another power-law conjecture appearing in statistical physics and combinatorial enumeration of clusters. Section A considers RN with site-dependent thresholds defined on graphs (Theorems A.2 - A.4). In Section 4 a brief discussion of the obtained results is presented.

\section{Rotators, networks, and avalanches}

In this section we define phase-shifting rotators, rotator networks, and avalanches. We formulate the Weyl theorem in uniform distribution on which our proofs are based and give a brief comparison of rotator networks with some other systems.

2.1. Phase-shifting rotator. The rotator networks that we consider consist of weakly interacting phase-shifting rotators (PSR). A rotator is a particle $P$ rotating on a circle - the phase variable $\Phi$ of $P$ (in polar coordinates with the origin at the center of the circle) is either increasing or a decreasing (depending on whether the rotation is clockwise or anti-clockwise) function of time $t$. In PSR two types of motion are allowed: uniform motion with constant rate and instantaneous rotations (shifts). The latter occur as a result of $\delta$-kicks: upon receiving a $\delta$-kick, a uniformly moving particle $P$ instantaneously shifts from its current position by a given small distance. The particle can be massive or massless, and we require that the particle's rate remain the same before and after such a shift. This means that the $\delta$-kicks are assumed to be not of mechanical character; particularly they must not be mixed with $\delta$-pulses (which affect the rate of a moving particle) considered in dynamical chaos (e.g., [16, 21]).

In what follows $h(t)$ is the Heaviside step-function: $h=0$ if $t \leq 0$ and $h=1$ otherwise; the notation $a=b \bmod (c)$ means: $a=b$ if $b<c$ and $a=c-b$ otherwise. The set $\mathbb{N}=\{1,2, \ldots\}$ is the natural series, $\mathbb{Z}$ is the set of all integers, $\mathbf{i}=\sqrt{-1}$ is the imaginary unit, $C=\left\{(2 \pi)^{-1} \exp (\mathbf{i} \Phi): 0 \leq \Phi \leq 2 \pi\right\}$ is the circle of unit length, and $I=[0,1]$ is the unit numerical segment. We assume that a label $\rho \in C$ or $\rho \in I$, which is understood as a threshold mark for some motion on $C$ or $I$, is assigned. We present a formal definition of PSR assigned specifically on circle $C$ or segment $I$. Then a PSR assigned on an arbitrary circle $C^{\prime}$ (or segment $I^{\prime}$ ) is then defined as follows: If $C$ and $C^{\prime}$ are found in complex plane $\mathbb{C}$ and the linear map $L(z)=a z+b$ is such that $L(C)=C^{\prime}$, a rotator $R^{\prime}$ on $C^{\prime}$ is defined as the composition $R^{\prime}=L^{-1} \circ R$ where $R$ is a rotator on $C$ (the same definition for the case of linear segments). For a finite set $e$ the $|e|$ denotes its power (the number of elements).

Definition 2.1. Let $\alpha$ and $\omega$ be some numbers, $\alpha \omega>0$, and $t_{n}>0$ be such that 
$t_{n+1}-t_{n}>\eta$ for some $\eta>0$ and all $n \in \mathbb{N}$. Let $k_{n} \in \mathbb{N}$ be bounded and $H$ be defined as

$$
H(t)=\omega t+\alpha \sum_{n \in \mathbb{N}} k_{n} h\left(t-t_{n}\right),
$$

where $t>0$ (time variable) and $h$ is the Heaviside function. A map of the form

$$
R: t \rightarrow(2 \pi)^{-1} \exp (\mathbf{i} H(t)) \quad \text { or } \quad R: t \rightarrow H(t) \quad \bmod (1)
$$

we call the phase-shifting rotator (PSR) assigned on $C$ (on I). The time series

$$
T^{(\text {in })}=\left\{t_{n}: n \geq 1\right\} \quad \text { and } \quad T^{(\text {out })}=\left\{\tau_{n}: R\left(\tau_{n}\right)=\rho\right\}
$$

are the input and output of the rotator $R$.

The $H(t)$ in (2.1) is the coordinate of a moving particle at time $t$ which starting at $t=0$ moves rectilinearly (or rotates on a circle, then $H(t)$ is proportional to the argument $\Phi(t)$ ) and uniformly with constant rate $\omega$ and at each moment $t_{n}$ is jumped along its orbit by a distance of length $|\alpha|$. As we mentioned, PSR must not be confused with the mechanically kicked rotator (e.g., $[16,21])$ : a massive particle rotating on a circle and at some moments receiving $\delta$-pulses from some periodic force. The mechanical rotator is a Hamiltonian system $([16,21])$ while PSR cannot be described in classical-mechanical terms. Indeed, a non-relativistic particle's rate just before and after any $\delta$-pulse cannot remain the same (see Section 1 ). Such a situation may happen only in relativistic theory when the particle's rate is close to speed of light e.g., a moving electron (in the framework of Bohr's atom model) receiving at some moments some quanta of energy $E=h \nu$.

2.2. Rotator networks and $\tau$-avalanches. Before giving a formal definition of rotator networks, we present their description using conventionally mechanical terminology. We consider a rotator network as a set of particles performing finite rectilinear motion on some segments; the "circular" interpretation of RN, dealing with rotating particles, is immediately derived if one identifies the end-points of these segments. The "linear" version of RN is more convenient in the proofs.

Let to every $i \in E_{N}$ a copy $I_{i}$ of unit segment $I=[0,1]$ and a particle $P_{i}$ moving on $I_{i}$ be given. The regular motion of $P_{i}$, when interaction with other particles is absent, is the following: when $P_{i}$ is found on $(0,1)$ it moves uniformly with the rate $\omega_{i}$ toward the threshold $\rho_{i}$ (for simplicity we assume $\rho_{i}=1$ if $\omega_{i}>0$ and $\rho_{i}=0$ otherwise); when reaching the threshold, $P_{i}$ transits instantaneously to the level $1-\rho_{i}$ and then starts again the same motion. Such a regular motion can be perturbed by some instantaneous shifts which occur at every moment $\left(=t_{n}^{(i)}\right)$ when some $k_{i} \geq 1$ $\left(k_{i}=k_{n}^{(i)}\right)$ particles from some sites $j \in E$ which are neighbor to $i$ simultaneously hit the levels $\rho_{j}$ (in what follows we count that $\alpha>0, \omega_{i}>0$ and all the thresholds $\rho_{i}$ are equal to 1 - this assumption does not affect on generality of results and their proof). Denoting by $H_{i}=H_{i}(t)$ the coordinate of $P_{i}$ on $I_{i}$, the motion of $P_{i}$ in this situation is described as follows: If $H_{i}+\alpha k_{i}<1$, the $P_{i}$ instantaneously lifts from its current position $H_{i}$ to $H_{i}+\alpha k_{i}$; and if $H_{i}+\alpha k_{i} \geq 1, P_{i}$ instantaneously drops from $H_{i}$ to $H_{i}+\alpha k_{i}-1$. Thus we distinguish two type of motions of the particle: the regular and instantaneous one; they can be expressed (respectively) as

$$
H_{i}(t)=\omega_{i} t \quad \bmod (1), \quad \text { and }\left(\text { for } t=t_{n}^{(i)}\right): H_{i}(t+0)=H_{i}(t)+\alpha k_{i} \quad \bmod (1) .
$$


One can see that at every moment $t$ the coordinate $H_{i}$ of $P_{i}$ is equal to

$$
H_{i}(t)=\omega_{i} t+\alpha \sum_{n \in \mathbb{N}} k_{n}^{(i)} h\left(t-t_{n}^{(i)}\right) \quad \bmod (1)
$$

where $h$ is Heaviside's function. As in Section 1, it is required that any shifting of a particle does not affect its rate, and the moments when a particle hits the corresponding threshold are separated by a positive constant.

According to Definition 2.1, for every $i \in E$ Equation (2.3) determines a PSR which we denote as $R_{i}$. In the described network the $\alpha$ in (2.3) does not depend on $i$ and the following linking condition holds: for every $i \in E_{N}$

$$
T_{i}^{(i n)}=\bigcup_{j \in \sigma(i)} T_{j}^{(o u t)}
$$

where it is assumed that for every term from $T_{i}^{(i n)}$, its multiplicity coincides with the number of its occurrences in different $T_{j}^{(o u t)}$ with $j \in \sigma(i)$. One can see that (2.4) is a necessary and sufficient condition for a set of PSR (having the same parameter $\alpha$ ) to be constituting some above-described network; i.e., the following definition can be given:

Definition 2.2. Let for every $i \in E_{N}$ a set $\sigma_{i} \subset E_{N}, i \notin \sigma_{i}$ be prescribed. A collection $\mathcal{R}=\left\{R_{1}, \ldots, R_{N}\right\}$ of (not necessary different) PSR with the same value of parameter $\alpha$ is called a rotator network (RN) assigned on $E_{N}$, if for every $i \in E_{N}$ condition (2.4) holds.

Despite the fact that (2.4) is a necessary requirement organizing a set of rotators into a network, this relation seems only of formal character and hardly suffices for computation of rotators' inputoutput series by the network's parameters $\bar{\omega}, \bar{\sigma}$, and $\alpha$. For that purpose the numerical method should rather be used: these series can be generated dynamically as the network is evolved with time $t$. We note that if a PSR is treated as a neuron, the output of every rotator in $\mathrm{RN}$ appears to be some analogy of neural spike trains of interacting neurons.

Our definition of avalanches involves a time parameter $\tau>0$ and thus differs from definitions accepted in SOC. To emphasize this we use the term $\tau$-avalanche. A $\tau$ avalanche consists of some single events whose precise occurrence times are found in the same time interval of length $\tau$.

Definition 2.3. Let $\mathcal{R}=\left\{R_{1}, \ldots, R_{N}\right\}$ be a rotator network. We say that at the $i$-th site at moment $t$ a single $\tau$-event occurs if the particle $P_{i}$ in $R_{i}$ hits the threshold $\rho_{i}$ during time interval $[t, t+\tau)$. The collection $A=A(t)$ of all single $\tau$-events which occur at the same moment $t$ is called a $\tau$-avalanche. The collection $S_{A}$ of all the sites at which a single $\tau$-event constituting a given $\tau$-avalanche $A$ occurs is called the support of $A$; a site of $S_{A}$ is called A-regular (A-singular) if the attainment of threshold at this site has resulted from regular (singular) motion. The number of vertices in $S_{A}$ is interpreted as the size or intensity of $A$. The duration of $A$ is the minimal time interval $T_{A}$ during which the series of all $\tau$-events constituting $A$ is started and then completed.

The $\tau$-avalanches in rotator networks can be studied in a rigorous way. To make this possible, one has to impose some restrictions on networks parameters; these are: the rate $\bar{\omega}$ of regular motion (clockwise rotation), the level of interaction $\alpha>0$, and 
time parameter $\tau$ through which the avalanches are defined. The restriction on the parameters is as follows: letting

$$
\beta=\frac{\max \left\{\omega_{i}: 1 \leq i \leq N\right\}}{\min \left\{\omega_{i}: 1 \leq i \leq N\right\}}-1, \quad \omega=N^{-1} \sum_{i=1}^{N} \omega_{i}, \quad \theta=\omega \tau
$$

we require that $\alpha, \beta$, and $\theta$ are small. It is also assumed that $\omega_{1}, \ldots, \omega_{N}$ are $\mathbb{Z}$ independent, i.e., for arbitrary $h_{i} \in \mathbb{Z}$ the relation $\sum_{i=1}^{N} h_{i} \omega_{i}=0$ implies that all the $h_{i}$ are zero; the collection of the $\bar{\omega}=\left(\omega_{1}, \ldots, \omega_{N}\right)$ with $\mathbb{Z}$-independent components is dense in Euclidean $\mathbb{R}^{N}$. In other words, our main results, which are of asymptotic character, are valid under the following conditions:

$$
\alpha+\beta+\theta \rightarrow 0, \quad \text { and } \omega_{1}, \ldots, \omega_{N} \text { are } \mathbb{Z} \text {-independent. }
$$

In what follows, two different situations, $\alpha / \theta=o(1)$ and $\theta / \alpha=o(1)$, are considered.

2.3. Weyl's theorem and some remarks. We formulate the Weyl theorem in uniform distribution (see, e.g., [14]) on which our proofs are based. Let $[x]$ denote the integer part of the real number $x$ (the maximal integer which is $\leq x),\{x\}=x-[x]$ be the fractional part of $x,\langle\mathbf{a}, \mathbf{b}\rangle=\sum_{k=1}^{N} a_{k} b_{k}$ be the inner product of vectors a and $\mathbf{b}$, i denote the imaginary unit, and vol and mes denote $N$-dimensional volume in $\mathbb{R}^{N}$ and Lebesgue measure on the real axis $\mathbb{R}^{1}$, respectively. Hereafter, for a vectorfunction $\mathbf{f}=\left(f_{1}, \ldots, f_{N}\right)$ we denote $\hat{\mathbf{f}}=\left(\left\{f_{1}\right\}, \ldots,\left\{f_{N}\right\}\right)$.

THEOREM 2.4. (H. Weyl) Let $\mathbf{f}(t)=\left(f_{1}(t), \ldots, f_{N}(t)\right)$ be a measurable vector-function, $\boldsymbol{\Delta} \subset \mathbb{D}^{N}$ be a finite union of $N$-dimensional parallelepipeds. If for every $\mathbf{h} \in \mathbb{Z}^{N}$ such that $\mathbf{h} \neq \mathbf{0}$,

$$
\lim _{T \rightarrow \infty} \frac{1}{T} \int_{0}^{T} \exp (2 \pi \mathbf{i}<\mathbf{h}, \mathbf{f}(t)>) d t=0
$$

then

$$
\lim _{T \rightarrow \infty} \frac{\operatorname{mes}(\{0 \leq t \leq T: \hat{\mathbf{f}}(t) \in \boldsymbol{\Delta}\})}{T}=\operatorname{vol}(\boldsymbol{\Delta})
$$

The orbits for which the latter equality holds are called ([14]) continuously uniformly distributed (c.u.d.) in $\mathbb{D}^{N}$. The next remark is a simple consequence (a particular case when $\mathbf{f}(t)$ is linear) of Weyl's theorem:

REMARK 2.5. Let $\omega_{1}, \ldots, \omega_{N}$ be $\mathbb{Z}$-independent, the numbers $a_{1}, \ldots, a_{N}$ be arbitrary, $\boldsymbol{\Delta}$ be a finite union of $N$-dimensional parallelepipeds from $\mathbb{D}^{N}$, and $\mathbf{S}$ be defined as $\mathbf{S}(t)=\left(a_{1}+\omega_{1} t, \ldots, a_{N}+\omega_{N} t\right)$. Then there exists the limit

$$
\lim _{T \rightarrow \infty} \frac{\{0 \leq t \leq T: \hat{\mathbf{S}}(t) \in \boldsymbol{\Delta}\}}{T}=m(\mathbf{S}, \boldsymbol{\Delta}), \quad \text { and } \quad m(\mathbf{S}, \boldsymbol{\Delta})=\operatorname{vol}(\boldsymbol{\Delta}) .
$$

The state space of rotator networks is either the $N$-dimensional cube (when we count that the particles $P_{i}$ move on linear segments) or the $N$-dimensional torus (if they rotate on circles). One can see that (due to a finite number of rotators in the 
network) the separation condition $t_{n+1}^{(i)}-t_{n}^{(i)} \geq \eta_{i}>0$ for inputs of rotators $R_{i}$ from a given network implies such a separation for their outputs $\tau_{n}^{(i)}$ :

REMARK 2.6. For every $R N$ there is a constant $\eta^{\prime}>0$ which depends only on $N$ and separation constants $\eta_{1}, \ldots, \eta_{N}$, such that $\tau_{n+1}^{(i)}-\tau_{n}^{(i)} \geq \eta^{\prime}$ for every $i \in E_{N}$ and for all $n \in \mathbb{N}$.

We say that time interval $\left[\tau_{n}^{(i)}, \tau_{n+1}^{(i)}\right]$ is the $n$-th cycle of motion of $P_{i}$. Let $k_{n}^{(i)}$ be the number of kicks received by $P_{i}$ from its neighbors during the $n$-th cycle. Every orbit of $\mathrm{RN}$ is of the form $\mathbf{H}_{\alpha}(t)=\left(\omega_{1} t+\alpha j_{1}(t), \ldots, \omega_{N} t+\alpha j_{N}(t)\right)$, where $j_{i}(t)=\sum_{0<t_{n}^{(i)} \leq t} k_{n}^{(i)}$ is the number of kicks received by $P_{i}$ during time $t$. The second sentence in the next remark is an obvious consequence of the first one; it follows from the separation condition for the input series in Definition 2.1.

REMARK 2.7. If $\alpha>0$ is small enough, then every particle $P_{i}$ during each cycle receives fewer than $c$ kicks, i.e., $0 \leq k_{n}^{(i)} \leq c$ for all $i$ and $n$; here $c>0$ is a constant which depends only on the separation constant $\eta$ but not on $i$ or $n$. Therefore, the orbit $\hat{\mathbf{H}}_{\alpha}(t)$ uniformly (in respect of $t \in[0,+\infty)$ ) converges (as $\alpha \rightarrow 0$ ) to some $\hat{\mathbf{S}}(t)$, and hence, for arbitrary $\Delta \subset \mathbb{D}^{N}$, the relation

$$
\lim _{\alpha \rightarrow 0} m\left(\mathbf{H}_{\alpha}, \boldsymbol{\Delta}\right)=m(\mathbf{S}, \boldsymbol{\Delta})=\operatorname{vol}(\boldsymbol{\Delta})
$$

holds.

The next remark reflects some features of $\tau$-avalanches; point (1) follows from (2.6) and Remark 2.7; the proof of point (2) is straightforward.

REMARK 2.8. (1) If $\alpha>0$ is small enough, then during every $\tau$-avalanche $A$ each particle on $S_{A}$ reaches threshold exactly once while no particle from the complement of $S_{A}$ reaches the threshold during this time interval. (2) If $S_{A}=S_{A^{\prime}}$ and $T_{A}=T_{A^{\prime}}$ then $A=A^{\prime}$; if $A \neq A^{\prime}$ then $T_{A} \cap T_{A^{\prime}}=\emptyset$.

2.4. Some comparisons. We present a brief comparison of RN (and related notions) with some other systems. The HHM, a neural network considered in [11, 12, 13] can be defined as follows: $0 \leq U_{i} \leq 1$ is the membrane voltage of the $i$-th neuron, the regular motion is the same as in (2.2) but with $\omega_{i} \equiv 1$, and the instantaneous motion is assigned by:

$$
U_{i}(t+0)=U_{i}(t)+\sum_{j} \alpha_{i, j}(t) \quad \text { or } \quad U_{i}(t+0)=\gamma\left(U_{i}(t)+\sum_{j} \alpha_{i, j}(t)-1\right),
$$

depending on whether the sum $U_{i}(t)+\sum_{j} \alpha_{i, j}(t)$ is less than 1 or not, (cp. Equation (2.3)); here, $0<\gamma \leq 1$ is a constant and $\alpha_{i, j}>0$ is understood as synaptic strength between the $i$-th and $j$-th neurons. If considering RN as neural networks, one should treat a PSR as a neuron, the $R(t)$ in Definition 2.1 as its current state, the output series $T^{(\text {out })}$ as a spike train, and the separation constant $\eta^{\prime}$ in Remark 2.6 as the neuron's refractory period. The BTW model supposes $\theta=\alpha=1 / 2 d$; this condition is intermediate between two limits, $\alpha / \theta=o(1)$ and $\theta / \alpha=o(1)$, assumed in the present work. There is an analog for our requirement $\alpha=o(1)$ (weak interaction) in HHM, which provides the convergence of its orbits [11] to some quasiperiodic [19] states. Point (1) in Remark 2.8 is not true for the BTW model but is essential in HHM. In $\mathrm{RN}$, the randomness (often postulated in different SOC systems) is substituted by 
$\mathbb{Z}$-independence (both are absent in HHM); due to the above-cited Weyl theorem; this enables us to reduce the computation of the frequency of the avalanches to a simpler geometrical task of computation of some volumes in the state space (see Remark 3.3). Every avalanche in the BTW model is an instantaneous event, and its support is always a connected set on the lattice; every $\tau$-avalanche in RN lasts a positive time, $0<\left|T_{A}\right| \leq \tau$, and its support can be an arbitrary set on the lattice (the latter holds also in HHM). It is possible in RN that a chain of different $\tau$-avalanches $A_{1}, A_{2}, \ldots$ immediately following each other will occur; in this case the supports of $A_{i}$ and $A_{i+1}$ are disjoint.

We present a (qualitative) comparison of rotator networks with the Turing-Smale mathematical model of morphogenesis. In his well-known work [22] Turing suggested the following system of ODEs

$$
\frac{d \mathbf{x}_{k}}{d t}=\mathbf{R}\left(\mathbf{x}_{k}\right)+\sum_{i} \mu_{i, k}\left(\mathbf{x}_{i}-\mathbf{x}_{k}\right) \quad(k=1, \ldots, N),
$$

which models a set of $N$ interacting biological cells; here $\mathbf{x}_{k} \in \mathbb{R}^{m}$ denotes the $k$-th cell, its components $\mathbf{x}_{k}^{j}$ denote the concentration of the $j$-th morphogen, $\mu_{i, k}$ are some $m \times m$ matrices, and the sum is taken over the neighbors of the $k$-th cell. In (2.11) the diffusion (transport of mass) from $\mathbf{x}_{i}$ to $\mathbf{x}_{k}$ arises when $\mathbf{x}_{i}^{j}>\mathbf{x}_{k}^{j}$ for some $1 \leq j \leq m$; in $\mathrm{RN}$, the $k$-th site receives a kick of positive intensity from its $i$-th neighbor when $H_{i}=1$ (and hence $H_{i} \geq H_{k}$ ). Smale [20] constructed some systems of the form (2.11), such that if $\mu_{i, k} \neq 0$, the system's orbits are asymptotically periodic, and if $\mu_{i, k}=0$ (i.e. the system without the sum in (2.11) that is responsible for interaction), they converge to some constants (the cells are inert); in rotator networks the orbits are of irregular character if the intensity of interaction $\alpha$ is nonzero and they are quasiperiodic if $\alpha=0$. In respect to the above-mentioned tendency to equalize the current states of the microsystems, we mention the difference analysis (see, e.g., [18]) where the dynamics of the equalizing process in application to discrete-time systems is studied.

Some modifications of the Turing-Smale have been suggested; e.g., we mention the well-known Kuramoto model of weakly coupled neural oscillators (see, e.g., [10]). A similar statement in dynamical networks, resulting from Huygens' earlier discovery on synchronization of almost identical coupled oscillators, is also a subject of many studies (see, e.g. [25]). The analytical results for synchronization of coupled threshold microsystems were obtained by several authors (see, [15] and references therein). In contrast, in the present work we are interested not in synchronization of the whole dynamics of coupled microsystems but only in their simultaneous (coherent) attaining of the threshold states on a given cluster ('firing of cluster'). Clearly, this is a different and less restrictive statement when different microsystems are each allowed to reach their thresholds in different ways (although again, almost simultaneously).

\section{Main results}

The theorems proved in this section establish the interrelation between the distribution of $\tau$-avalanches in rotator networks and the network's combinatorial characteristics. Following traditions in the SOC and the BK model, and in order to simplify our statement, we define and consider the networks on Euclidean lattices. In Section A we briefly explain how the results obtained are extended to rotator networks on graphs with site-dependent values of thresholds. Before presenting our main theorems we need to introduce some definitions and formulate some remarks.

As before we denote by $|S|$ the power (number of entries) of the set $S, E_{N}=$ $[1, \ldots, N]$, and let $\mathcal{R}=\left\{R_{1}, \ldots, R_{N}\right\}$ be a rotator network on $E_{N}$. Let also $\mathcal{S}=\{S\}$, 
$\mathcal{S} \subset 2^{E_{N}}$ be some (arbitrary) collection of subsets of $E_{N}$. We are interested in computation of the frequency of $\tau$-avalanches having a given support $S \subset E_{N}$ as well as in computation of the distribution of $\tau$-avalanches whose supports belong to a given collection $\mathcal{S}$. These are defined as follows:

Definition 3.1. Let $S \subset E_{N}$ and $\mathcal{S}=\{S\}$ be a collection of subsets of $E_{N}$. The frequency of avalanches with the support $S$ and the distribution of avalanches with the supports from a given $\mathcal{S}$ are defined as

$$
\pi_{S}=\lim _{T \rightarrow \infty} \frac{\operatorname{mes}(e(S, T))}{T} \quad \text { and } \quad \pi_{\mathcal{S}}(k)=\sum_{S \in \mathcal{S},|S|=k} \pi_{S} \quad(k=1,2, \ldots, N)
$$

respectively; here $1 \leq k \leq N$ and $e(S, T)$ denotes the set of all the moments $1 \leq t \leq T$ at which a $\tau$-avalanche with the support $S$ occurs.

In what follows in this section we consider RN assigned on Euclidean $d$ dimensional lattices $\mathbb{L}^{d}=L \times \cdots \times L \quad(d$ times $)$; here $L=[1, \ldots, m]$ is the segment of $m$ integers, $d \geq 1$ and $m \geq 2$. The lattice $\mathbb{L}^{d}$ consists of $N=m^{d}$ vertices (sites, nodes), which are numbered arbitrarily, $\mathbb{L}^{d}=\left\{v_{1}, v_{2}, \ldots, v_{N}\right\}$. We consider connected sets (clusters, lattice animals, LA) on the lattice $\mathbb{L}^{d}$ : a set $S \subset \mathbb{L}^{d}$ is called connected if for every pair of different vertices $u, v \in S$ there exists a series $u_{0}, \ldots, u_{n}$ of vertices from $S$ such that $u_{0}=u, u_{n}=v$, and for all $i=1, \ldots, n$ the Euclidean distance between $u_{i-1}$ and $u_{i}$ is equal 1 . Every non-empty set on the lattice can be represented in the form of the union of some pairwise disjoint clusters (connected components of $S$ ).

The "nearest-neighbor" (in the sense of Euclidean metric) relation on the lattice $\mathbb{L}^{d}$ determines some special neighborhood $\bar{\sigma}=\left(\sigma_{1}, \ldots, \sigma_{N}\right)$ on $E_{N}(=[1, \ldots, N]): j \in$ $\sigma_{i}$ whenever the Euclidean distance between $v_{i}$ and $v_{j}$ is equal 1. Every network $\mathcal{R}=\left\{R_{1}, \ldots, R_{N}\right\}$ of rotators on $E_{N}$ having such a special $\bar{\sigma}$ can be considered as a network assigned on $\mathbb{L}^{d}$ : the rotator $R_{i}$ is now assigned not to the index $i \in E_{N}$ but to the site $v_{i} \in \mathbb{L}^{d}$ (the same substitution made in Definition 3.1 for avalanches on the sets $S \subset \mathbb{L}^{d}$ ). The circumstance which allows the application of the above cited Weyl's theorem is the following: every $\tau$-avalanche can be geometrically interpreted as a parallelepiped in the state space of the network. To explain this point some additional notions are required:

Definition 3.2. Let $S \subset \mathbb{L}^{d}$ be a non-empty set and $C=\left(S_{0}, S_{1}\right)$ be a partition of $S$ into two disjoint subsets $S_{0}$ and $S_{1}: S_{0} \cup S_{1}=S, S_{0} \cap S_{1}=\emptyset, S_{0} \neq \emptyset$ such that every connected component of $S$ contains at least one vertex from $S_{0}$ and for any vertex from $S_{0}$ all its neighbors on the lattice, which belong to $S$, are found in $S_{1}$. Every such partition $C$ of $S$ we call a color of $S$ assuming that the vertices from $S_{0}$ are colored into red and vertices from $S_{1}$ are colored into blue. The collection of all such colors $C$ of $S$ is denoted $\mathcal{C}(S)$.

In this definition the requirements for the distribution of red and blue are not symmetric: e.g., if a partition $C$ of a non-empty set $S$ contains only one red vertex while all the others in $S$ (if any) are blue then $C \in \mathcal{C}(S)$, but if a $C$ contains only one blue vertex in $S$ and all the others in $S$ are red then $C \notin \mathcal{C}(S)$. For example, if each connected component of a given $S$ contains exactly one red vertex while all the others in $S$ are blue, then such a partition belongs to $\mathcal{C}(S)$. The following provides another example of considering colors: if a partition $C$ of $S$ is such that for every red vertex $v \in S$ all its neighbors belonging to $S$ are blue and for every blue vertex $v \in S$ there is a red vertex in $S$ which is a neighbor to $v$, then $C \in \mathcal{C}(S)$. 
Let $S \subset \mathbb{L}^{d}$ be non-empty set, $C \in \mathcal{C}(S)$, and vectors $\mathbf{k}=\left(k_{1}, \ldots, k_{N}\right)$ and $\bar{\delta}=$ $\left(\delta_{1}, \ldots, \delta_{N}\right)$ be such that $k_{n} \in \mathbb{N}, 1 \leq k_{n} \leq N, k_{n} \alpha<1$ and components of $\bar{\delta}$ are small. With every such $C, \bar{\delta}$, and $\mathbf{k}$ we associate an $N$-dimensional parallelepiped $B_{C}(\bar{\delta}, \mathbf{k})$ from $\mathbb{D}^{N}$ defined as: $B_{C}(\bar{\delta}, \mathbf{k})=J_{1} \times \cdots \times J_{N}$, where the intervals $J_{n} \subset[0,1]$ are

$$
J_{n}= \begin{cases}\left(0,1-m \alpha-\delta_{n}\right), & v_{n} \in M_{S}^{(m)}, \\ \left(0,1-\delta_{n}\right), & v_{n} \in \mathbb{L}^{d} \backslash \bar{S} \\ \left(1-\delta_{n}, 1\right), & v_{n} \in S_{0} \\ \left(1-k_{n} \alpha, 1\right), & v_{n} \in S_{1}\end{cases}
$$

here, $M_{S}$ is the border of $S$ (the collection of the vertices from $\mathbb{L}^{d} \backslash S$ which contain at least one neighbor belonging to $S), M_{S}^{(m)}$ is the collection of all sites of $M_{S}$ each of which possesses exactly $m$ neighbors belonging to $S$, and $\bar{S}=S \cup M_{S}$. Let us denote $\theta_{i}=\omega_{i} \tau$ and $B_{C, \mathbf{k}}=B_{C}\left(\theta_{1}, \ldots, \theta_{N} ; \mathbf{k}\right)$. Since for every $\tau$-avalanche $A$ the collections of all $A$-regular and $A$-singular vertices constitute a color from $\mathcal{C}\left(S_{A}\right)$ (see Definition 2.3 ; the regular vertices are red and singular ones are blue), every $\tau$-avalanche with support $S$ determines some $B_{C, \mathbf{k}}$. Taking into account Remark 2.7 and point (1) of Remark 2.8, one can see that the following is true:

REMARK 3.3. Let $S \subset \mathbb{L}^{d}$ be a non-empty set and $A$ be a $\tau$-avalanche with the support $S$. There exists a color $C \in \mathcal{C}(S)$ and a vector $\mathbf{k}$ such that $\mathbf{S}_{\alpha}(t) \in B_{C, \mathbf{k}}$ if and only if $t \in T_{A}$.

Our next formulations involve some combinatorial characteristics — with a given $S \subset \mathbb{L}^{d}$ we associate two polynomials: for a vector $\mathbf{q}=\left(q_{1}, \ldots, q_{N}\right)$ where $1 \leq q_{n} \leq N$ we let

$$
G_{S}^{(0)}(\alpha, \theta, \mathbf{q})=\sum_{n \in \mathbb{N}} a_{0}(n, S)\left(\theta / q_{n} \alpha\right)^{n}, \quad G_{S}^{(1)}(\alpha, \theta, \mathbf{q})=\sum_{n \in \mathbb{N}} a_{1}(n, S)\left(q_{n} \alpha / \theta\right)^{n},
$$

where $a_{\delta}(n, S)(\delta \in\{0,1\})$ denotes the total number of partitions $\left(S_{0}, S_{1}\right) \in \mathcal{C}(S)$ for which $\left|S_{\delta}\right|=n$. In what follows, the notation $\varphi \sim \psi$ means $\varphi=\psi+o(\psi)$.

LEMMA 3.4. Let $\mathcal{R}$ be a rotator network on $\mathbb{L}^{d}$ and $S$ be an arbitrary set on the lattice $\mathbb{L}^{d}$. Under conditions (2.6), for asymptotic frequency $\pi_{S}$ of $\tau$-avalanches in $\mathcal{R}$ with the support $S$ the relations

$$
\pi_{S} \sim(q \alpha)^{|S|} G_{S}^{(0)}(\alpha, \theta, \mathbf{q}) \quad(\alpha / \theta \rightarrow 0) \quad \text { and } \quad \pi_{S} \sim \theta^{|S|} G_{S}^{(1)}(\alpha, \theta, \mathbf{q}) \quad(\theta / \alpha \rightarrow 0)
$$

with some $\mathbf{q}$ and $1 \leq q \leq N$ hold.

Proof. In order to simplify the notation we assume $\tau=1$. It follows from the first condition in (2.6) that

$$
\operatorname{vol}\left(\bigcup_{C \in \mathcal{C}(S)} B_{C, \mathbf{q}}\right) \sim \operatorname{vol}\left(\bigcup_{C \in \mathcal{C}(S)} B_{C}^{\prime}\right)
$$

where $B_{C}^{\prime}$ denotes $B_{C}^{\prime}=B_{C}(\theta, \ldots, \theta ; \mathbf{q})$ and $\theta$ is defined in $(2.5)$; therefore, due to Remark 2.7 and Remark 3.3, $\pi_{S}$ converges (as $\alpha+\beta+\theta \rightarrow 0$ ) the to $N$-dimensional volume

$$
\lambda_{S}=\operatorname{vol}\left(\bigcup_{C \in \mathcal{C}(S)} B_{C}^{\prime}\right)
$$


Since $\alpha, \beta$, and $\theta$ are small, from the definition in Equ. (3.2) it follows that

$$
\operatorname{vol}\left(B_{C}^{\prime}\right) \sim k_{C}^{\left|S_{1}\right|} \theta^{\left|S_{0}\right|} \alpha^{\left|S_{1}\right|} \quad \text { where } \quad 1 \leq k_{C} \leq N \quad\left(k_{C}=\left(\prod_{n=1}^{\left|S_{1}\right|} q_{n}\right)^{1 /\left|S_{1}\right|}\right) .
$$

Using the inclusion-exclusion procedure:

$$
\operatorname{vol}\left(\bigcup_{C \in \mathcal{C}(S)} B_{C}^{\prime}\right)=\sum_{p \geq 1}(-1)^{p-1} \sum_{1 \leq i_{1}<i_{2}<\cdots<i_{p} \leq r} \operatorname{vol}\left(B_{C_{i_{1}}}^{\prime} \cap B_{C_{i_{2}}}^{\prime} \cap \cdots \cap B_{C_{i_{p}}}^{\prime}\right)
$$

(it is assumed that $\mathcal{C}(S)$ is numbered, $\mathcal{C}(S)=\left\{C_{1}, C_{2}, \ldots, C_{r}\right\}$ ), we obtain

$$
\operatorname{vol}\left(\bigcup_{C \in \mathcal{C}(S)} B_{C}^{\prime}\right) \geq \sum_{C \in \mathcal{C}(S)} \operatorname{vol}\left(B_{C}^{\prime}\right)-\sum_{1 \leq i_{1}<i_{2} \leq r} \operatorname{vol}\left(B_{C_{i_{1}}}^{\prime} \cap B_{C_{i_{2}}}^{\prime}\right) \text {. }
$$

Equations (3.2) and (3.4) imply that for $i \neq j$

$$
\operatorname{vol}\left(B_{C_{i}}^{\prime} \cap B_{C_{j}}^{\prime}\right) \leq \min \left\{\frac{\theta}{\alpha}, \frac{\alpha}{\theta}\right\} \min \left\{\operatorname{vol}\left(B_{C_{i}}^{\prime}\right), \operatorname{vol}\left(B_{C_{j}}^{\prime}\right)\right\} ;
$$

consequently, for the last sum in (3.5) we obtain

$$
\sum_{1 \leq i_{1}<i_{2} \leq r} \operatorname{vol}\left(B_{C_{i_{1}}}^{\prime} \cap B_{C_{i_{2}}}^{\prime}\right)=\sum_{i=1}^{r} \sum_{j=1}^{r-i} \operatorname{vol}\left(B_{C_{i}}^{\prime} \cap B_{C_{i+j}}^{\prime}\right) \leq \min \left\{\frac{\theta}{\alpha}, \frac{\alpha}{\theta}\right\} r \sum_{C \in \mathcal{C}(S)} \operatorname{vol}\left(B_{C}^{\prime}\right),
$$

and then it follows from Equations (3.3), (3.4), and (3.5) that

$$
\lambda_{S}=(1+o(1)) \sum_{C \in \mathcal{C}(S)} \operatorname{vol}\left(B_{C}^{\prime}\right)
$$

where the term $o(1)$ is as small as we like. Using (3.4) one can see that Lemma 3.4 is proved.

The following speculation (which then results in Theorem 3.5) leads to two numerical characteristics of subsets $S \subset \mathbb{L}^{d}$ in terms of which the relations in Lemma 3.4 can be given in a simpler form: since $G_{S}^{(\delta)}(\delta \in\{0,1\})$ is a polynomial, its growth is determined by two numbers $\mu_{\delta}(S)$ and $\nu_{\delta}(S)$ :

$$
G_{S}^{(0)}(\alpha, \theta, \mathbf{q}) \sim \nu_{0}(S)(\theta / q \alpha)^{\mu_{0}(S)}, \quad G_{S}^{(1)}(\alpha, \theta, \mathbf{q}) \sim \nu_{1}(S)(q \alpha / \theta)^{\mu_{1}(S)},
$$

where $q=q_{S}$ and $1 \leq q \leq N$. It follows from definition of the coefficients $a_{\delta}$, that $\mu_{\delta}$ and $\nu_{\delta}$ can also be defined without referring to these polynomials: $\mu_{\delta}(S)=\max \left\{\left|S_{\delta}\right|\right.$ : $\left.\left(S_{0}, S_{1}\right) \in \mathcal{C}(S)\right\}$ and $\nu_{\delta}(S)$ is the number of $(\delta)$-maximal colors of $S$ (a color of $S$ is called $(\delta)$-maximal if $\left.\left|S_{\delta}\right|=\mu_{\delta}(S)\right)$.

The next theorem, which is an immediate consequence of Lemma 3.4 and relations (3.6), expresses the $\pi_{S}$ explicitly through the characteristics $\mu_{\delta}$ and $\nu_{\delta}$ of the $S$ and parameters $\alpha$ and $\theta(=\omega \tau)$. If interpreting the RN as neural networks, one may treat a given set $S$ on the lattice as some pattern and the avalanches that occur over the $S$ as the way for its dynamical generation; then Theorem 3.5 allows us to control the pattern's appearance probability (by varying the parameters $\alpha$ or $\theta$ ). As before, in the following formulations the notation $\varphi \sim \psi$ means $\varphi=\psi+o(\psi)$. 
THEOREM 3.5. Let $\mathcal{R}$ be a rotator network on $\mathbb{L}^{d}$ and $S$ be a set on the lattice $\mathbb{L}^{d}$. Under conditions (2.6), the asymptotic frequency $\pi_{S}$ of $\tau$-avalanches in $\mathcal{R}$ with the support $S$ can be computed by the formulas

$$
\begin{aligned}
\pi_{S} & \sim \nu_{0}(S)(q \alpha)^{|S|}(\theta / q \alpha)^{\mu_{0}(S)} \quad \text { if } \quad \alpha / \theta \rightarrow 0, \\
\pi_{S} & \sim \nu_{1}(S) \theta^{|S|}(q \alpha / \theta)^{\mu_{1}(S)} \quad \text { if } \quad \theta / \alpha \rightarrow 0 ;
\end{aligned}
$$

here $q=q_{S}$ and $1 \leq q \leq N$.

Theorem 3.5 somewhat contradicts (due to the presence of the coefficients $\nu_{0}$ and $\nu_{1}$ in (3.7) and (3.8)) some assumptions in combinatorics ([24]) according to which the probability associated with a LA depends on its size but not configuration. In LA-related problems arising in statistical physics (e.g., [8]) and combinatorics (e.g., [24]) one usually deals with some collection $\mathcal{S}$ of subsets of $\mathbb{Z}^{d}$ which is specified by some criteria. For example, $\mathcal{S}$ can be required to consist of percolation or Laplacian clusters (cell growth statement in statistical physics, [8]), convex clusters (enumeration in combinatorics), unions of some clusters, etc. We consider $m$-chains on $\mathbb{L}^{d}(1 \leq m \leq$ $d)$ : such a chain is defined as a cluster which is isomorphic to an $m$-dimensional parallelepiped from $\mathbb{Z}^{d}$ (we say that $S$ and $S^{\prime}$ are isomorphic if there exists a one-toone map $S \rightarrow S^{\prime}$ preserving the neighborhood relation). The 1-chain is a zigzag line, and 2-chain is a two-dimensional serpentine-like folded surface on $\mathbb{L}^{d}$. The chains are the simplest clusters, but sometimes they can be of most interest in applications (e.g., see Section 4). Obviously, if $S$ and $S^{\prime}$ are isomorphic then $\mu_{\delta}(S)=\mu_{\delta}\left(S^{\prime}\right)$ and $\nu_{\delta}(S)=\nu_{\delta}\left(S^{\prime}\right)$ - for some clusters this circumstance reduces the computation of these characteristics to computation of ones for a simpler configuration. For example, for arbitrary 1- and 2-chains $S \subset \mathbb{L}^{d}$ we obtain $\mu_{\delta}(S)=[(|S|+1) / 2]$ (since this holds for linear segments and rectangles).

Let us formulate a consequence of Theorem 3.5 which for some special collections $\mathcal{S}$ gives us an asymptotic expression for distribution $\pi_{\mathcal{S}}$. We call $\mathcal{S}$ isometric if for arbitrary $S, S^{\prime} \in \mathcal{S}$ the relation $|S|=\left|S^{\prime}\right|$ implies that $S$ and $S^{\prime}$ are isomorphic; for instance, for each $m$ the collection of $m$-chains is isometric. For every isometric $\mathcal{S}$ there exist two functions $\varphi_{\delta}$ and $\psi_{\delta}$ of natural argument (we do not study their explicit form) such that $\mu_{\delta}(S)=\varphi_{\delta}(|S|), \nu_{\delta}(S)=\psi_{\delta}(|S|)$, and the term $q=q_{S}$ in Theorem 3.5 depends only on the power $|S|$. From (3.7) and (3.8) it follows that:

THEOREM 3.6. Let $\mathcal{R}$ be a rotator network on $\mathbb{L}^{d}$ and $\mathcal{S}$ be an isometric collection on $\mathbb{L}^{d}$. Under conditions (2.6) the distribution $\pi_{\mathcal{S}}$ of $\tau$-avalanches in $\mathcal{R}$ with supports from $\mathcal{S}$ can be computed by the formulas

$$
\begin{aligned}
& \pi_{\mathcal{S}}(k) \sim \alpha^{k} \psi_{0}(k)(\theta / q \alpha)^{\varphi_{0}(k)} N_{\mathcal{S}}(k) \quad \text { if } \quad \alpha / \theta \rightarrow 0, \\
& \pi_{\mathcal{S}}(k) \sim \theta^{k} \psi_{1}(k)(q \alpha / \theta)^{\varphi_{1}(k)} N_{\mathcal{S}}(k) \quad \text { if } \quad \theta / \alpha \rightarrow 0 ;
\end{aligned}
$$

here $q=q_{k}, 1 \leq q \leq N$, and $N_{\mathcal{S}}(k)$ is the number of entries of $\mathcal{S}$ of size $k$.

For every cluster $S \subset \mathbb{Z}^{2}$ its (0)-maximal color can be constructed as follows: there exist exactly two partitions $\left(\mathbb{Z}_{\epsilon}^{2}, \mathbb{Z}_{1-\epsilon}^{2}\right)(\epsilon=0,1)$ of $\mathbb{Z}^{2}$ such that for every vertex from $\mathbb{Z}_{\epsilon}^{2}$ all its neighbors belong to $\mathbb{Z}_{1-\epsilon}^{2}$; one of them (or both) induces a (0)-maximal color of $S$ (we omit the strict proof). For a set $S \subset \mathbb{L}^{d}, S=\bigcup_{i=1}^{\varkappa} S_{i}$, and $S_{i}$ are some pairwise disjoint clusters its every (1)-maximal partition consists of a single red vertex 
in each $S_{i}$, while all other vertices (if any) in $S_{i}$ are blue (in particular, this yields the equality in (3.12) and the following relation: $\left.\nu_{1}(S)=\prod_{i=1}^{\varkappa}\left|S_{i}\right|\right)$. We estimate $\mu_{\delta}$ and $\nu_{\delta}$ as follows:

REMARK 3.7. For every $S \subset \mathbb{L}^{d}$ the relations

$$
\begin{aligned}
& \lambda_{d, 1}|S| \leq \mu_{0}(S) \leq \lambda_{d, 2}|S|, \quad 1 \leq \nu_{0}(S) \leq 2^{|S|}, \\
& \mu_{1}(S)=|S|-\varkappa, \quad|S| / \varkappa \leq \nu_{1}(S) \leq(|S| / \varkappa)^{\varkappa},
\end{aligned}
$$

where $\lambda_{d, 1}=\frac{1}{2 d+1}, \lambda_{d, 2}=\frac{2 d}{2 d+1}$, and $\varkappa$ is the number of connected components of $S$, hold.

Proof. To prove (3.11) we first assume that $S$ is a cluster and let its partition $\left(S_{0}, S_{1}\right)$ be (0)-maximal. Since for every $v \in S_{0}$ all its neighbors belong to $S_{1}$, we have $\left|S_{0}\right| \leq 2 d\left|S_{1}\right|$. Since $S_{0}$ is (0)-maximal, it follows that every $v \in S_{1}$ possesses at least one neighbor belonging to $S_{0}$, and, consequently, $\left|S_{1}\right| \leq 2 d\left|S_{0}\right|$. These two imply the sought inequalities for $\mu_{0}(S)$. If $S=\bigcup_{i} S_{i}$ is an arbitrary set (and $S_{i}$ are its pairwise disjoint connected components) then $\mu_{0}(S)=\sum_{i} \mu_{0}\left(S_{i}\right)$, and the sought inequalities follow from the ones for the clusters $S_{i}$. In (3.11) the upper bound for $\mu_{0}$ is the exact one: it is assumed, e.g., for clusters consisting of a single vertex with all its $2 d$ neighbors. The inequality for $\nu_{0}$ in $(3.11)$ is obvious, since $2^{|S|}$ is the number of arbitrary colorings of the elements of $S$ into red and blue (more precise estimates are also possible; e.g., since $\nu_{0}(S) \leq\left(\begin{array}{c}|S| \\ \mu_{0}\end{array}\right)$, using Stirling's formula one can obtain: $\nu_{0}(S) \leq 2^{c|S|}$ for some $0 \leq c<1$ and large enough $\left.|S|\right)$. The second relation in (3.12) follows from equalities $\left|S_{1}\right|+\cdots+\left|S_{\varkappa}\right|=|S|$ and $\nu_{1}(S)=\prod_{i=1}^{\varkappa}\left|S_{i}\right|$ : the geometrical mean of the numbers $\left|S_{i}\right|$ is less than the arithmetical one, and since $\left|S_{i}\right| \geq 1$, at least one term in the above sum is larger than the arithmetical mean. Remark 3.7 is proved. 口

Let us consider the situation when $\mathcal{S}$ consists of arbitrary subsets of $\mathbb{L}^{d}$, i.e., no special restriction on its terms is imposed. The next theorem connects the distribution $\pi_{\mathcal{S}}$ of $\tau$-avalanches with the combinatorial distribution of $\mathcal{S}$. We consider two such distributions (using the same notation): $N_{\mathcal{S}}(k)$ is the number of entries of $\mathcal{S}$ of size $k$ and $N_{\mathcal{S}}(k, \varkappa)$ is the number of entries of $\mathcal{S}$ of size $k$ having $\varkappa$ connected components. As before, in the next formulation the $\theta$ is defined as $\theta=\omega \tau$.

THEOREM 3.8. Let $\mathcal{R}$ be a rotator network on $\mathbb{L}^{d}$ and $\mathcal{S}$ be a collection of subsets of $\mathbb{L}^{d}$. Under conditions (2.6) the distribution $\pi_{\mathcal{S}}$ of $\tau$-avalanches in $\mathcal{R}$ with the supports from $\mathcal{S}$ can be computed by formulas

$$
\begin{array}{llr}
\pi_{\mathcal{S}}(k) \sim\left(c_{1}(q \alpha)^{c_{2}} \theta^{1-c_{2}}\right)^{k} N_{\mathcal{S}}(k) & \text { if } & \alpha / \theta \rightarrow 0, \\
\pi_{\mathcal{S}}(k) \sim \alpha^{k} \sum_{\varkappa=1}^{k}(k / \varkappa)^{c_{k}, \varkappa}(\theta / q \alpha)^{\varkappa} N_{\mathcal{S}}(k ; \varkappa) & \text { if } & \theta / \alpha \rightarrow 0 ;
\end{array}
$$

here $q=q_{k}, 1 \leq q \leq N$, the $c_{1}$ and $c_{2}$ which may depend on $k$ are such that $1 \leq c_{1} \leq 2$, $\lambda_{d, 1} \leq c_{2} \leq \lambda_{d, 2}$, and $1 \leq c_{k, \varkappa} \leq \varkappa$.

Proof. This theorem follows from Theorem 3.5 almost immediately. Indeed, taking into account Remark 3.7, Equation (3.7) can be rewritten as

$$
\pi_{S} \sim\left(c_{1}(S)(q \alpha)^{c_{2}(S)} \theta^{1-c_{2}(S)}\right)^{k}
$$


where $k=|S|$, and for every $S$ the coefficients $c_{1}(S)$ and $c_{2}(S)$, depending only on dimensionality $d$, are found within the same bounds as in the formulation of the theorem. For each $k$ the relation in the left hand side of (3.15) is a continuous function in variables $c_{1}$ and $c_{2}$, defined in the rectangle $D \subset \mathbb{R}^{2}$ determined by inequalities from the formulation of the theorem. Its average on a finite set of pairs $\left(c_{1}(S), c_{2}(S)\right.$ ) (where $S \in \mathcal{S}$ and $|S|=k)$ from $D$ is found between its minima and maxima in $D$; since the average is always assumed at some point of $D$, (3.15) implies (3.13). To prove (3.14) we note that it follows from Theorem 3.5 and Equ. (3.12) that for every $S$ having $\varkappa$ connected components we obtain

$$
\pi_{S} \sim(k / \varkappa)^{c(S)} \alpha^{k}(\theta / q \alpha)^{\varkappa},
$$

where $1 \leq c(S) \leq \varkappa$. Arguing as above, we obtain Equ. (3.14). Theorem 3.8 is proved.

(

If $\mathcal{S}$ consists of clusters, Theorem 3.8 links the computation of the distribution of $\tau$-avalanches with the problem of combinatorial enumeration of LA. In an abstract setting the latter problem is to compute the number of LA on a lattice some of whose parameters (e.g., size, perimeter, etc.) satisfy certain limitations. There are many results on enumeration, related to computer and computation theory, molecular biology, and statistical physics (see, e.g., [24] and references therein). In statistical physics the LA appear in connection with critical behavior in lattice models of such phenomena as crack propagation, electrical discharge, percolation, etc ([8]). To our knowledge, the main conjecture in this field $([5,8,9]$ and $[23,24])$ :

$$
N(k) \sim \text { const. } c^{k} k^{-\lambda_{2}} \quad\left(c>1, \quad \lambda_{2}>0\right),
$$

where $N(k)$ is the number of non-congruent LA from $\mathbb{Z}^{2}$ of size $k$, still remains unresolved. As an example, we present some of such results (the notation $N(k)$ now stands for the total number of corresponding clusters): for oriented 1-chains in $\mathbb{Z}^{d}$ and 1-chains in $\mathbb{Z}^{2}: N(k)=d^{k}$ and $N(k)=(1+\sqrt{2})^{k}-(1-\sqrt{2})^{k}([17])$; for "real" 1 chains in $\mathbb{Z}^{2}$ and $\mathbb{Z}^{3}: N(k)=c^{k} k^{1 / 3}$ and $N(k)=c^{k} k^{1 / 6}$ ([9]); for convex clusters in $\mathbb{Z}^{2}$ with perimeter $2 k: N(2 k+8)=(2 k+11) 4^{k}-4(2 k+1)\left(\begin{array}{c}2 k \\ k\end{array}\right)([5])$.

One can deal only with avalanches whose supports are non-congruent; e.g., one can be interested in the dynamical appearance (i.e., as the supports of some avalanches) of some configurations (e.g., rectangles, circle-like objects, some images) ignoring their precise position on the lattice. Such assumption on non-congruency of LA, appearing in different models of fractal growth, is present (though implicitly) in many works in statistical physics (e.g., [8]). Particularly, this concerns models of electric discharge and crack-propagation ([8]), whose mechanism is close to ones using in modelling the seismicity. This is, the collection $\mathcal{S}$ in the theorems above is assumed to be such that it does not contain two different congruent items. In this situation, assuming that the linear size $L$ of $\mathbb{L}^{2}$ is large enough (then one can assume that all the non-congruent LA from $\mathbb{Z}^{2}$ of relatively small power are found on $\mathbb{L}^{2}$ ), we compare the conjectures (1.1) and (3.16): for relatively (in respect to $L$ ) small $k$ Equation (3.13) in Theorem 3.8 implies that for some values $\lambda, c$ (in (1.1) and (3.16)) and parameters $\alpha$ and $\theta$ the two conjectures are almost equivalent: if we accept (1.1) (or (3.16)), Theorem 3.8 yields a relation which almost coincides with the other conjecture (3.16) (or (1.1)). More precisely, the following is true:

Corollary 3.9. In rotator networks (with $\alpha / \theta=o(1)$ ) on $\mathbb{L}^{2}$, conjecture (1.1) is equivalent to conjecture (3.16) where the coefficient $c$ is allowed to have some (arbitrarily slow) growth to $+\infty$ (and then it follows that $\lambda_{1}=\lambda_{2}$ ). 


\section{Conclusions}

The results presented assert that in rotator networks. $N_{\mathcal{S}}$, which is defined as the combinatorial distribution of a given collection $\mathcal{S}$, strongly correlates with the dynamical distribution $\pi_{\mathcal{S}}$ of $\tau$-avalanches whose supports belong to $\mathcal{S}$. Thus, if $\mathcal{S}$ consists of clusters (connected sets), then according to our theorems $N_{\mathcal{S}}$ should be recognized as the network's basic connectivity-related characteristic affecting its dynamics (avalanche distribution). The theorems presented contain some answer to the following question: if one can register the avalanches in the network, then what conclusion can be drawn about the network's architecture? And conversely, knowing the network's structural data, what can be said about the avalanches in the network?

The features of the networks reflected by $N_{\mathcal{S}}$ and $\pi_{\mathcal{S}}$ are in a certain sense opposite: we illustrate this on the following example. Let the network consists of neurons $x \in X$, and let $U_{x}(t)$ be the membrane voltage of neuron $x$ at moment $t$. For integrateand-fire neurons the $U_{x}$ can be (roughly) treated as a rotator: $U_{x}(t)$ increases, when reaching the threshold it drops to a level close to initial one, and a firing of the neuron occurs.

It is claimed in neuroscience that in terms of coherent firings (avalanches) of neurons the basic information-coding mechanism could be expressed. Therefore, while $N_{\mathcal{S}}$ seems to be of a technological character (wiring), the $\pi_{\mathcal{S}}$ should rather be classified according to system's information-processing capabilities. Some discussions on this matter can be found in $[11,12,13]$ (avalanches in neural nets) and in [3] (connectivity in the brain; e.g., it is known that the cerebellum consists of 1-, 2-, and 3-chains). The question of how these two opposite entities relate to each other and in what terms their correlation should be expressed is of the prior interest in brain research. In this work we introduced weakly coupled pulse working systems, the networks of phase-shifting rotators, which are close to neural nets and which permit some answer to this question.

Appendix A. Generalized rotator networks. In this section we generalize the rotator networks in such a way that the rotators may possess arbitrary values of thresholds but theorems from previous Section 3 remain valid. Moreover, since in the proofs in Section 3 the Euclidean structure (e.g., metric, linear independence) of the lattices $\mathbb{L}^{d}$ has not been taken into account, in this section we consider the rotator networks assigned on graphs, where the connectivity (neighborhood relation) is the only structure.

Let $X$ be a finite set and $\sigma: X \rightarrow 2^{X}(x \notin \sigma(x))$ be a map prescribing to every $x \in X$ a subset $\sigma(x) \subset X$ (the pair $(X, \sigma)$ is an oriented graph). The map $\sigma$ assigns a neighborhood on $X$ : entries of $\sigma(x)$ are understood as neighbors for $x$. Assuming reflexivity of $\sigma$, i.e., $x \in \sigma(y)$ whenever $y \in \sigma(x)$, the clusters (as well as $m$-chains and isometric collections) can be defined; particularly, every $S \subset X$ can be represented in the form of a union of pairwise disjoint clusters. Let $X$ be arbitrarily numbered, $X=\left\{x_{1}, x_{2}, \ldots, x_{N}\right\}$ and with the $i$-th node $x_{i}$ a (generalized) rotator $R_{i}$ be prescribed. A (generalized) rotator consists of a particle $P$ rotating with a constant angular rate $\omega$ on a circle $C$ with radius $r>0$ (and a threshold mark $\rho \in C$ ) which at some moments $t_{n}$ separated by a positive constant receives some $\delta$-kicks of small constant intensity. That is, the $H$ from Definition 2.1 gains the form

$$
H(t)=\omega r t+\alpha r \sum_{n \in \mathbb{N}} k_{n} h\left(t-t_{n}\right),
$$

where $\alpha$ is the intensity of $\delta$-kicks, and $t_{n}$ satisfies the same separation condition as 
in Definition 2.1. Descriptively, Equ. (A.1) means that at each moment $t\left(=t_{n}^{(i)}\right)$ when the particle $P_{i}$ in $R_{i}$ hits the threshold $\rho_{i}$, it sends a $\delta$-kick to each $P_{j}$ whose index $j$ is in $\sigma(i)$; this kick results in instantaneous rotation of $P_{j}$ on the angle $\alpha$. In the "linear" case, when particles perform motion on some segments $I_{i}$ of length $r_{i}$ (for simplicity one may assume that $I_{i}=\left[0, r_{i}\right]$ and $\rho_{i}=0$ ), upon receiving a $\delta$-kick the $P_{j}$ instantaneously shifts by the distance $\alpha r_{j}$ : If $H_{j}+\alpha r_{j}<r_{j}$ the $P_{j}$ lifts from its current position $H_{j}$ to $H_{j}+\alpha r_{j}$; and if $H_{j}+\alpha r_{j} \geq r_{j}$, the $P_{j}$ drops from $H_{j}$ to the level $H_{j}+\alpha r_{j}-r_{j}$. This is, interaction in the system is essentially the same as before: we accept the micro-dynamics as in (2.3) (or (2.2) but replacing $\bmod$ (1) by $\bmod \left(r_{i}\right)$ ). The generalized $\mathrm{RN}$ is defined as a network $\mathcal{R}=\left\{R_{1}, \ldots, R_{N}\right\}$ of such generalized rotators $R_{i}$ for which the corresponding functions $H_{i}$ are of the form (A.1); it is also assumed that the linking condition Equ. (2.4) holds.

Let us explain how the linear transform $L(z)=a z+b$ determines (in accordance with definitions in Section 2.1) the PSRs assigned on circles of arbitrary radius. Let (as in Section 2.1) $C \subset \mathbb{C}$ (the complex plane) be a circle with center $z=0$ and length 1 and $P$ be a particle rotating on $C$ with angular rate $\omega$, receiving $\delta$-kicks of intensity $\alpha$ at some moments $t_{n}$. Let $C^{\prime}$ be a concentric circle with radius $r=|a| / 2 \pi$ and $P^{\prime}$ be the intersection of $C^{\prime}$ with the ray emerging from $z=0$ and passing through $P$. Then $P^{\prime}$ rotates on $C^{\prime}$ with the same angular rate $\omega$ and receives $\delta$-kicks of intensity $\alpha$ at the same moments $t_{n}$. Thus a PSR assigned on $C$ determines some PSR on $C^{\prime}$. Shifting $C^{\prime}$ on vector $b$ we obtain a rotator assigned on the circle with center $b$ and radius $|a| / 2 \pi$.

The assumption that the absolute value $\left(=r_{i}\right)$ of the threshold may vary with the site influences the form of some previous limitations and let us clarify this point. The interaction in the system is assigned as in (2.3) (or (2.2) but replacing mod (1) by $\left.\bmod \left(r_{i}\right)\right)$. The state space of the network is now either the parallelepiped $\mathbb{P}=\left[0, r_{1}\right] \times$ $\cdots \times\left[0, r_{N}\right]$ or torus $\mathbb{T}=C_{1} \times \cdots \times C_{N}$. Given a vector-function $\mathbf{f}(t)=\left(f_{1}(t), \ldots, f_{N}(t)\right)$ we let

$$
\tilde{\mathbf{f}}=\left(r_{1}^{-1} f_{1}, \ldots, r_{N}^{-1} f_{N}\right), \quad \hat{\mathbf{f}}=\left(r_{1}\left\{r_{1}^{-1} f_{1}\right\}, \ldots, r_{N}\left\{r_{N}^{-1} f_{N}\right\}\right)
$$

and for $\boldsymbol{\Delta} \subset \mathbb{P}$ define $e(\mathbf{f}, \boldsymbol{\Delta}, T)=\{0<t<T: \hat{\mathbf{f}}(t) \in \boldsymbol{\Delta}\}$; we say that $\mathbf{f}$ is c.u.d. in $\mathbb{P}$ if for an arbitrary union of parallelepipeds $\boldsymbol{\Delta} \subset \mathbb{P}$

$$
\lim _{T \rightarrow \infty} \frac{m e s(e(\mathbf{f}, \boldsymbol{\Delta}, t))}{T}=\frac{\operatorname{vol}(\boldsymbol{\Delta})}{\operatorname{vol}(\mathbb{P})} .
$$

Instead of the $\mathbb{Z}$-independence of $\omega_{1}, \ldots, \omega_{N}$ we now require the $\mathbb{Z}$-independence of the numbers $\omega_{1} r_{1}, \ldots, \omega_{N} r_{N}$. To prove the next remark it suffices to take into account Remark 2.7 and apply the cited Weyl's theorem to the vector-function $A \tilde{\mathbf{f}}$, where $A$ is the linear map $A: \mathbb{P} \rightarrow \mathbb{D}^{N}$ defined by the diagonal matrix $\left[a_{i, j}\right]_{i, j=1}^{N}$ with $a_{i, i}=r_{i}$ :

REMARK A.1. (1) A vector-function $\mathbf{f}$ is c.u.d. in the parallelepiped $\mathbb{P}$ iff $\tilde{\mathbf{f}}$ is c.u.d. in $\mathbb{D}^{N}$; (2) If $\omega_{1} r_{1}, \ldots, \omega_{N} r_{N}$ are $\mathbb{Z}$-independent, then $\mathbf{f}(t)$ is c.u.d. in $\mathbb{P}$.

The $\tau$-avalanches in such generalized networks, their frequencies and distributions, as well as the other notions (such as $C(S), \mu_{\delta}, \nu_{\delta}$, clusters, connected components, etc.) appeared in the previous formulations of theorems and proofs and are defined in the same way as before. The next theorems are valid under the following limitations:

$$
\alpha+\beta+\theta \rightarrow 0, \quad \text { and } \quad \omega_{1} r_{1}, \ldots, \omega_{N} r_{N} \text { are } \mathbb{Z} \text {-independent }
$$


where $\alpha, \beta$ and $\theta$ are the same as in Equ. (2.5) (since the angular rates of $P$ and $P^{\prime}$ in the above-mentioned concentric rotators are the same); their proof remains the same as for the corresponding theorems in Section 3. That is, the following three theorems, where the same two situations $-\alpha / \theta=o(1)$ and $\theta / \alpha=o(1)$ - are considered, are true:

TheOREM A.2. Let $\mathcal{R}$ be a rotator network on $X$ and $S$ be a subset of $X$. Under conditions (A.2) the asymptotic frequency $\pi_{S}$ of $\tau$-avalanches in $\mathcal{R}$ with the support $S$ can be computed by Equations (3.7) and (3.8).

THEOREM A.3. Let $\mathcal{R}$ be a rotator network on $X$ and $\mathcal{S}$ be an isometric collection on $X$. Under conditions (A.2) the distribution $\pi_{\mathcal{S}}$ of $\tau$-avalanches in $\mathcal{R}$ with supports from $\mathcal{S}$ can be computed by Equations (3.9) and (3.10).

TheOREM A.4. Let $\mathcal{R}$ be a rotator network on $X$ and $\mathcal{S}$ be collection of arbitrary subsets of $X$. Under conditions (A.2) the distribution $\pi_{\mathcal{S}}$ of $\tau$-avalanches in $\mathcal{R}$ with supports from $\mathcal{S}$ can be computed by Equations (3.13) and (3.14).

Acknowledgement. The authors thank the referees of this work, whose remarks and suggestions allowed us to improve the manuscript. A.S. would like to thank R. Hilfer, V.B. Priezzhev, and D. Dhar for useful discussions. This research was partially supported by the Heisenberg-Landau Program.

\section{REFERENCES}

[1] G. Adomian, The Burridge-Knopoff model, J. App. Math. Comp., 77(2-3), 131-132, 1996.

[2] P. Bak, C. Tang and K. Weisenfeld, Self-organized criticality: an explanation of $1 / f$ noise, Phys. Rev. Lett., 59, 381-384, 1987.

[3] V. Braitenberg, Information from structure: a sketch of neuroanatomy, in Models of Neural Networks, I, E. Domany, J.L. van Hemmen and K. Schulten (Eds), Springer, Berlin, 1994.

[4] R. Burridge and L. Knopoff, Model and theoretical seismicity, Bull. Seis. Soc. Am., 57, 341-371, 1967.

[5] M.P. Delest and G. Viennot, Algebraic languages and polyominoes enumeration, Theor. Comput. Sci., 34, 169-206, 1984.

[6] D. Dhar, Self-organized critical state of sandpile automaton models, Phys. Rev. Lett., 64, 1613-1616, 1990.

[7] D. Dhar, Exact solution of a directed-site animals enumeration problem in 3 dimensions, Phys. Rev. Lett., 59, 853-856, 1983.

[8] C.W. Lung, Fractals in Physics, Proc. VI-th Int. Trieste Conf., Eds: L. Pietronero and E. Tosatti, ICTP, Trieste, Italy, 1985.

[9] P.G. de Gennes, Scaling Concepts in Polymer Physics, Ithaca, 1976.

[10] F.C. Hoppensteadt and E.M. Izhikevich, Oscillatory neurocomputers with dynamic connectivity, Phys. Rev. Lett., 82, 2983-2986, 1999.

[11] A.V. M. Herz, Global analysis of recurrent neural networks, in Models of Neural Networks, III, E. Domany, J.L. van Hemmen and K. Schulten (Eds), Springer, Berlin, 1994.

[12] A.V. M. Herz and J.J. Hopfield, Rapid local synchronization of action potentials: toward computation with coupled integrate-and-fire neurons, Proc. Natl. Ac. Sci., 92, 6655-662, 1995.

[13] A.V. M. Herz and J.J. Hopfield, Earthquake cycles and neural reverberations: collective oscillations in systems with pulse-coupled threshold elements, Phys. Rev. Lett., 75, 1222-1225, 1995.

[14] L. Kuipers and H. Niederreiter, The Uniform Distribution of Sequences, Wiley, 1976.

[15] R.E. Mirrolo and S.H. Strogatz, Synchronization of pulse-coupled biological oscillators, SIAM J. Appl. Math., 50, 1645-1662, 1999.

[16] H.G. Schuster, Deterministic Chaos, Physik-Verlag, Weinheim, 1984.

[17] A. Yu. Shahverdian, Lattice animals and self-organized criticality, Fractals, 5, 199-213, 1997.

[18] A. Yu. Shahverdian and A.V. Apkarian, A difference characteristic for one-dimensional deterministic systems, Comm. Nonlin. Sci. and Comp. Sim., 12, 233-242, 2007.

[19] W. Singer, The role of synchrony in neocortical processing, in Models of Neural Networks, II, E. Domany, J.L. van Hemmen and K. Schulten (Eds), Springer, Berlin, 1994. 
[20] S. Smale, A mathematical model of interaction of two cells using the Turing's equation, in The Hopf Bifurcation and Applications, J.E. Marsden, M. McCracken, Springer-Verlag, New York, 1976.

[21] H.J. Stockmann, Quantum Chaos, Cambridge Univ. Press, 2000.

[22] A.M. Turing, The chemical basis for morphogenesis, Phil. Trans. Roy. Soc., (B), 37-72, 1925.

[23] G. Viennot, Enumeration combinatories and algebraic languages, Lect. Notes in Comput. Sci., Fundamentals of Comput. Theory, L. Budach (Eds), 1985.

[24] G. Viennot, Problemes combinatoires poses par la Physique statistique, Seminaire Bourbaki, n626, 36eme annee, in Asterique, Soc. Math. France, n(121-122), 225-246, 1985.

[25] D.J. Watts and S.H. Strogatz, Collective dynamics of 'small-world' networks, Nature, June, 393, 440-442, 1998. 